\title{
USING IMPROVED RADIAL BASIS FUNCTIONS METHODS FOR FLUID-STRUCTURE COUPLING AND MESH DEFORMATION
}

\author{
Giorgos A. Strofylas ${ }^{1}$, Georgios I. Mazanakis ${ }^{1}$, Sotirios S. Sarakinos ${ }^{1}$, Georgios N. Ly- \\ gidakis $^{1}$ and Ioannis K. Nikolos ${ }^{2}$
}

\author{
Technical University of Crete, School of Production Engineering and Management \\ University Campus, Chania, GR-73100, Greece \\ ${ }^{1}$ e-mail: \{gstrofylas, gmazanakis, ssarakinos, glygidakis\}@isc.tuc.gr \\ 2 e-mail: jnikolo@dpem.tuc.gr
}

Keywords: Fluid-Structure Interaction, Radial Basis Functions, Information Transfer, Mesh Deformation, Non-matching meshes, Partitioned Coupling.

\begin{abstract}
In this work the development of a partitioned FSI coupling procedure is reported, aiming to facilitate interaction between an open-source CSD (Computational Structural Dynamics) and an in-house academic CFD (Computational Fluid Dynamics) code. Attention is mainly directed towards the efficient and accurate transfer of predicted displacements, velocities (by CSD) and loads (by CFD). More precisely, spatial coupling is achieved using Radial Basis Functions (RBFS) interpolation, which enables point-based interaction, needing therefore no information for connectivities and, consequently, allowing for the utilization of different type or even intersecting structural and flow grids. Although RBFs method seems to be particularly attractive for both data transfer and mesh deformation, it suffers from a significant drawback; it calls for relatively excessive memory and computation time requirements (in its initial formulation). In case of data transfer the Partition of Unity (PoU) approach is adopted as a remedy of the aforementioned deficiency, which regards the decomposition of the examined problem into several smaller ones, to be solved independently and hence more efficiently. In mesh deformation though, improvement of computational performance is succeeded with a surface point reduction technique, based on the agglomeration of the adjacent boundary nodes, i.e., on the fusion of the RBFs centers. Despite the notable reduction of RBFs base points, the proposed method preserves sufficiently the quality of the initial grid. The proposed algorithm is evaluated against a benchmark (for FSI solvers) test case, considering the analysis of the wind action over a standard tall building model. The obtained numerical results confirm its potential for such simulations, highlighting additionally the radically improved computational performance of data transfer and grid deformation procedures.
\end{abstract}




\section{INTRODUCTION}

During the past decades the analysis of Fluid-Structure-Interaction (FSI) with numerical methods gained significant popularity, mainly due to the broad range of its applications, e.g., in aerodynamic shape optimization, aeroelastic analysis, etc. Although several FSI solvers, commercial, industrial or academic, have already been developed, the need for more accurate and more efficient methodologies is still on the run, making FSI a continuously evolving scientific field. One of the most widely applied techniques to deal with this kind of problems is the partitioned coupling approach. According to this method the fluid and structural physical systems are solved independently, while interaction between them is obtained via the exchange of appropriate variables on boundary surfaces [1,2]; as a result, it allows for the integration of separately developed CSD (Computational Structural Dynamics) and CFD (Computational Fluid Dynamics) applications. Nevertheless, the aforementioned solvers' combination is raising several issues in terms of information transfer, considering the main task of accurate as well as efficient FSI simulations.

For the numerical solution of FSI problems, the Dirichlet-Neumann domain decomposition coupling approach is usually followed [3]. It begins with the flow prediction, while at next the obtained pressure field at the flow side of the CFD/CSD interface is transferred to the corresponding structural one. Subsequently, a CSD analysis is performed, deriving the deflections of the structure and a new flow grid is generated incorporating the new interface location. Information transfer across the fluid-structure interface has to be performed with respect to a kinematic condition (Dirichlet), assuring the continuity of the velocity field, and a dynamic condition (Neumann) satisfying the requirement for conservation of normal stresses [4]. Hence, the most important criteria to be fulfilled over the interface, concern the consistency between the structural and the flow solver and the instabilities that may occur due to the partitioning process as well as the strategy followed for the data transfer. Similarly to stresses' conservation, an energy one has to be succeeded at the common interface [1] to ensure the stability of the whole numerical solution, i.e., the virtual work of the flow forces and this of the structural ones must be equal. The aforementioned conservation requirements across the fluid-structure interface depend strongly on both the employed temporal and spatial coupling schemes [5].

This study focus is mainly towards the spatial coupling and information transfer between the CFD and CSD solvers. The computational nodes of the structural and flow grids do not coincide usually at their interfaces, since their underlying resolutions can significantly differ. This nonmatching interface may lead to gaps and intersections between them, necessitating thus for an extra interpolation step to enable information transfer. Numerous methods can be found in the literature to deal with this problem, varying from the simplistic neighbor interpolation method [6] to more sophisticated approaches, such as the weighting ones [7, 8]. More elaborate attempts include the use of projections and integrations of elements from one mesh to the other [9], while popular among researchers are the Constant Volume Tetrahedra [10,11] and the Boundary Element (BEM) [12] methods. In this work information transfer is achieved via an interpolation scheme based on Radial Basis Functions (RBF) [13, 14], the appealing properties of which have been extensively analyzed by Beckert and Wendland in [15], e.g., the ability to inherently conserve total force and moment. RBFs methodology has another significant advantage; it does not require any connectivity information, as it relies only on arbitrary point data. As a result, it can cope both with structured and unstructured grids.

Apart from the data transfer, in FSI problems a new volume flow mesh has to be generated, depending on the boundary displacements and, consequently, on the new interface location; this procedure is typically implemented several times during an FSI simulation, necessitating thus 
to be versatile, efficient, and accurate, as well as to sufficiently preserve the initial mesh topology [16]. A considerably appealing method for mesh deformation is based on the extension of RBFs interpolation, used to transfer data across the interface, in the context of mesh motion [16]. Nevertheless, the method, in its initial formulation, calls for relatively excessive memory and computation time requirements. For the alleviation of the aforementioned shortcoming the Partition of Unity (PoU) [17] approach is adopted in data transfer, which regards the decomposition of the interfacing boundary into several smaller regions, where local interpolations are performed independently and hence more efficiently. Although this method ensures energy conservation and offers physical distribution of the forces on the interfacing area $[17,18]$, it is revealed to be inappropriate for mesh deformation, as the dependence matrix takes into account all the flow mesh nodes, entailing significantly increased computational cost . In order to mitigate this inadequacy, a relatively recently developed by the authors methodology is followed, considering agglomeration of the surface nodes, and as such reduction of the dimensions of the pre-mentioned matrix [19]; it is based on the fusion strategy, developed for a corresponding CFD agglomeration multigrid method [20]. The method achieves significant acceleration of the deformation procedure, without downgrading the quality of the derived meshes [19].

In this work a partitioned FSI coupling procedure was developed to facilitate interaction between an open-source CSD [21] and an in-house academic CFD [20] code. Attention is mainly directed toward the efficient and accurate transfer of predicted displacements (by CSD) and forces (by CFD). Therefore, spatial coupling is obtained by a RBFs interpolation scheme, employing the aforementioned PoU approach [18] to accelerate the exchange procedure. For mesh deformation a corresponding RBFs interpolation process is applied; its efficiency is significantly improved with a surface point reduction technique, based on the agglomeration of adjacent boundary nodes. The proposed algorithm is evaluated against a benchmark (for FSI solvers) test case, considering the analysis of the wind action over a standard tall building model $[22,23]$. The obtained numerical results confirm its potential for such simulations, highlighting additionally the radically improved computational performance of data transfer and grid deformation procedures.

The rest of the paper is organized as follows: In Section 2 the coupling procedure, concerning exchange of displacements and forces, is described in detail, while in Section 3 the incorporated mesh deformation process is outlined. Section 4 includes a brief description of the flow and structural models. In Section 5 the validation results of the proposed methodology over a benchmark test case are presented, while Section 6 contains some conclusions, based on the aforementioned results.

\section{COUPLING PROCEDURE}

The spatial coupling procedure can be mainly divided in two stages, namely, (a) the transfer of the displacement vectors from the structural to the flow grid and (b) the transfer of nodal forces from the flow mesh back to the structural one. The coupling scheme, for both aforementioned steps, can be expressed in a unified fashion by defining a coupling matrix $H$, which associates the interface boundary nodes of the two grids (flow and structural). Then, interpolation of the displacements can be performed according to:

$$
d_{s}=H d_{f}
$$

where, $d_{s}$ and $d_{f}$ denote the displacements at the structural and flow interface side, respectively. For forces' association, the transpose matrix $H^{\mathrm{T}}$ is used as

$$
f_{s}=H^{\mathrm{T}} f_{f},
$$


where $f_{s}$ and $f_{f}$ are the forces acting on the structural and flow sides of the interface, respectively. With the adoption of this scheme the virtual work can be effectively conserved over the interface $[24,25]$; nevertheless, the conservation of total force and moment depends mainly on the choice of the coupling matrix $H$, and hence on the corresponding method applied to obtain it.

\subsection{Radial Basis Functions (RBFs) interpolation}

In this work, the RBFs technique is implemented to construct the interpolation matrix, which is used for exchanging information between the structural and the flow solver. According to the RBFs theory [15], the interpolation function is defined as a weighted sum of the evaluations of the basis functions:

$$
s(\boldsymbol{x})=\sum_{i=1}^{n_{c}} a_{\mathbf{i}} \Phi\left(\left\|\boldsymbol{x}-\boldsymbol{x}_{\boldsymbol{c}_{\boldsymbol{i}}}\right\|\right)+p(\boldsymbol{x}),
$$

where $a_{i}$ are the weight coefficients, $p(x)$ a polynomial, and $\boldsymbol{x}_{\boldsymbol{c}_{\boldsymbol{i}}}=\left[x_{c_{i}}, y_{c_{i}}, z_{\mathrm{i}}\right]$ the RBFs centers, e.g., the coordinates of the boundary mesh nodes; $n_{c}$ is the number of those centers and $\Phi$ is the basis function. The use of the linear polynomial term ensures the exact recovery of translations and rotations [15]. The polynomial term in $x$ direction can be expressed as follows

$$
p^{x}\left(\boldsymbol{x}_{c_{i}}\right)=\gamma_{0}^{x}+\gamma_{1}^{x} x+\gamma_{2}^{x} y+\gamma_{3}^{x} z
$$

while the coefficients $a_{i}$ and $\gamma_{i}$ are computed by interpolation conditions, fulfilling Equation (3) requirements at the centers of the basis functions, along with an additional condition, described as

$$
\begin{gathered}
s\left(\boldsymbol{x}_{c_{i}}\right)=\boldsymbol{U}_{c_{i}}=\left[u_{c_{i}}^{x}, u_{c_{i}}^{y}, u_{c_{i}}^{z}\right]^{T}, \\
\sum_{\mathrm{i}=1}^{n_{c}} a_{i} q\left(\boldsymbol{x}_{\boldsymbol{c}_{\boldsymbol{i}}}\right)=0,
\end{gathered}
$$

where $\boldsymbol{U}_{c_{i}}$ are the known boundary displacements of the structural side of the interface.

Several examples of different basis functions can be found in the literature $[13,15,26]$, which can be broadly classified to functions with global and local support. In the first case the functions influence the whole domain, while in the latter ones the variable to be interpolated is scaled with a support radius $R(\xi=x / R)$, indicating the region of influence of the function from each center [26]. Moreover, it should be noted that smoother basis functions result in more realistic deformations, which in turn provoke dense matrix systems entailing numerical instability. In this work Wendland's $C^{2}$ basis function with local support radius was adopted, due to its robustness and accuracy [16].

To construct the coupling matrix $H$, the structural mesh displacements of the boundary surfaces are used; combining Equations (5) and (6), the coefficient vectors $\boldsymbol{a}^{\boldsymbol{x}, \boldsymbol{y}, \boldsymbol{z}}$ and $\boldsymbol{b}^{\boldsymbol{x}, \boldsymbol{y}, \boldsymbol{z}}$ can be evaluated by solving the following linear system in each spatial direction (expressed in matrix notation in the $x$ direction)

$$
\left[\begin{array}{c}
U_{\mathrm{x}} \\
0
\end{array}\right]=\left[\begin{array}{cc}
\boldsymbol{M}_{c c} & \boldsymbol{P}_{c} \\
\boldsymbol{P}_{c}^{T} & 0
\end{array}\right]\left[\begin{array}{l}
a^{x} \\
b^{x}
\end{array}\right]=C_{c c}\left[\begin{array}{l}
a^{x} \\
b^{x}
\end{array}\right]
$$

where

$$
\boldsymbol{a}^{x}=\left[\begin{array}{c}
a_{c_{1}}^{x} \\
a_{c_{2}}^{x} \\
\vdots \\
a_{c_{n}}^{x}
\end{array}\right], \quad \boldsymbol{b}^{x}=\left[\begin{array}{c}
\gamma_{0}^{x} \\
\gamma_{1}^{x} \\
\gamma_{2}^{x} \\
\gamma_{3}^{x}
\end{array}\right]
$$

and 


$$
\boldsymbol{P}_{\boldsymbol{c}}=\left[\begin{array}{cccc}
1 & x_{1} & y_{1} & z_{1} \\
1 & x_{2} & y_{2} & z_{2} \\
\vdots & \vdots & \vdots & \vdots \\
1 & x_{\mathrm{c}_{n}} & y_{\mathrm{c}_{n}} & z_{\mathrm{c}_{n}}
\end{array}\right], \boldsymbol{M}_{\boldsymbol{c c}}=\left[\begin{array}{cccc}
\Phi_{11} & \Phi_{12} & \cdots & \Phi_{1 \mathrm{c}_{n}} \\
\Phi_{21} & \Phi_{22} & \cdots & \Phi_{2 \mathrm{c}_{n}} \\
\vdots & \vdots & \vdots & \vdots \\
\Phi_{\mathrm{c}_{n} 1} & \Phi_{\mathrm{c}_{n} 2} & \cdots & \Phi_{\mathrm{c}_{n} \mathrm{c}_{n}}
\end{array}\right]
$$

with $\Phi_{i j}=\Phi\left(\left\|x_{i}-x_{j}\right\|\right)$.

The required approximation of displacements at the flow side of the interface are calculated by applying the following equation for each spatial direction:

$$
s\left(x_{a}\right)=\boldsymbol{A}_{a c} \boldsymbol{C}_{c c}^{-1} x_{c}=\boldsymbol{H} x_{c}
$$

where

$$
\boldsymbol{A}_{a c}=\left[\begin{array}{cccccccc}
1 & x_{\alpha_{1}} & y_{\alpha_{1}} & z_{\alpha_{1}} & \Phi_{\alpha_{1} c_{1}} & \Phi_{\alpha_{1} c_{2}} & \cdots & \Phi_{\alpha_{1} c_{n}} \\
1 & x_{\alpha_{2}} & y_{\alpha_{1}} & z_{\alpha_{1}} & \Phi_{\alpha_{2} c_{1}} & \Phi_{\alpha_{2} c_{2}} & \cdots & \Phi_{\alpha_{2} c_{n}} \\
\vdots & \vdots & \vdots & \vdots & \vdots & \vdots & \vdots & \vdots \\
1 & x_{\alpha_{N_{\alpha}}} & y_{\alpha_{N_{\alpha}}} & z_{\alpha_{N_{\alpha}}} & \Phi_{\alpha_{N_{\alpha} c_{1}}} & \Phi_{\alpha_{N_{\alpha}} c_{2}} & \cdots & \Phi_{\alpha_{N_{\alpha}} c_{n}}
\end{array}\right],
$$

with $a_{i}$ being the $i$-th flow mesh node of the boundary and $N_{\alpha}$ the total number of boundary nodes of the same grid. If the following term is additionally set

$$
\tilde{M}_{c c}=\left(P_{c} M_{c c}^{-1} P_{c}^{T}\right)^{-1}
$$

then the coupling matrix $H$ can be expressed as:

$$
\boldsymbol{H}=\boldsymbol{A}_{a c}\left[\begin{array}{c}
\widetilde{M}_{c c} P_{c} M_{c c}^{-1} \\
M_{c c}^{-1}-M_{c c}^{-1} P_{c}^{T} \widetilde{M}_{c c} P_{c} M_{c c}^{-1}
\end{array}\right]
$$

Following the coupling process described above, it is evident that the computation of the coupling matrix is obtained by solving the linear systems described in Equation (7), while the interpolation of displacements and forces from the one mesh to the other involve only matrixvector multiplications. However, the method suffers from excessive computational and memory requirements, especially when large-scale problems are encountered, since the coupling matrix $\boldsymbol{H}$ is of size $N_{s} \mathrm{x} N_{\alpha}$, where $N_{s}$ denotes the number of the structural mesh surface nodes and $N_{\alpha}$ the number of the flow mesh surface nodes. Computational efficiency is further degraded due to the necessity to invert the $\left(n_{c}+4\right) \mathrm{x}\left(n_{c}+4\right)$ matrix $\boldsymbol{C}_{c c}$. A thorough literature review of methods that deal with the aforementioned deficiency can be found in [19]; the boundary node coarsening $[19,27,28]$ technique seems to be particularly attractive for the mesh deformation process (as it will be analyzed in the next section). However, it is considered inappropriate for the data transfer one, as it might result in loss of information and, as such, jeopardize the requirement of force conservation over the interface.

Another approach, also focusing on the efficiency improvement of the method, is the Partition of Unity (PoU) scheme, according to which the initial problem is divided into several smaller ones by grouping base points into sets to be processed separately; it resembles the domain decomposition approach, employed for the parallelization of complex numerical methods. It has been successfully implemented against FSI problems in $[17,18]$ with very promising results. Besides reducing computational cost, it avoids the undesirable influence of support radius into the interpolation process.

\subsection{Partition Of Unity (PoU)}

The basic concept of the PoU method regards the degradation of the initial problem into several smaller ones to be solved independently, while the global solution arises by a weighted 
combination of the local ones. Given this condition, the term "unity" refers to the summation of the weighting coefficients of the entire domain that must be equal to one. The process begins by dividing the set $\Omega$ of the boundary nodes, including the nodes of the interface of both computational meshes (structural and flow) into a $K$ number of mutually overlapping regions, in a way to ensure that each $\Omega_{j}$ patch includes only a small number of points. For each of these patches a local displacement is computed by solving a local interpolation problem with the RBFs method. Subsequently, the global interpolation is obtained by summing all local interpolations, weighted by their respective coefficients, as

$$
s(\boldsymbol{x})=\sum_{j=1}^{K} w_{j}(\boldsymbol{x}) s_{j}(\boldsymbol{x}), \quad \sum_{j=1}^{K} w_{j}(\boldsymbol{x})=1,
$$

where $w_{j}(\boldsymbol{x})$ represents a non-negative weighting function, which comes into effect only for its associated patches $\Omega j$, while it is zeroed for the rest. Using the transpose of the global matrix, formed by the local interpolants, assures that the conservation criteria are also met in the PoU approach [17].

A variation of the pre-described method, proposed by Rendall and Allen [18], concerns the application of the PoU method on each boundary flow node separately, instead of decomposing the set of nodes into overlapping patches. Following this strategy, the complex step of decomposing the structural grid into overlapping regions is evaded, and thus the possibility of facing discontinuities between the patches due to the partitioning process is diminished. In order to calculate the local interpolants, an association of each flow mesh node with a pre-defined number of its closest structural boundary ones has to be defined. Therefore, the use of sophisticated data structures appears to be mandatory in order to link the boundary nodes of the two grids (flow and structural) in an efficient manner. Octree data structures are a common tactic for addressing these kind of problems. After the definition of the local interpolants, their merging back to a global one is essential in order to avoid distortion and non-realistic deformations, which may occur in applications involving computational meshes with different resolutions. In addition, the implementation of such a local strategy ameliorates the force distribution over the interface; the area of influence of each flow node is limited only to its nearby ones of the structural grid, unlike the corresponding area used with the complete method.

\section{MESH DEFORMATION}

The formulation of RBFs in case of mesh motion is different from this of data transfer, since the two problems have different requirements. In case of data transfer the inclusion of polynomial terms of at least first order is mandatory to ensure conservation of total force and moment, while for mesh deformation these functions are omitted to avoid its undesirable global coverage, which can cause the whole domain to move rather than deform. Hence, the dependence matrix $\boldsymbol{C}_{c c}$ is reduced to $\boldsymbol{M}_{c c}$, and the weighting coefficients are obtained via the following expression

$$
\boldsymbol{a}^{x}=\boldsymbol{M}_{c c}^{-1} \boldsymbol{x}_{c_{i}},
$$

with $\boldsymbol{a}^{x, y, z}$ denoting the vector containing the scalar coefficients in each direction and $\boldsymbol{M}_{\boldsymbol{c c}}$ the matrix defined in Equation (9).

The required displacements of the volume mesh nodes are calculated according to those of the boundary points. As such, the deformation of the volume mesh can be computed as follows

$$
\boldsymbol{s}\left(x_{a}\right)=\boldsymbol{A}_{c v} \boldsymbol{M}_{c c}^{-1} \boldsymbol{x}_{c_{i}}=\boldsymbol{H} \boldsymbol{x}_{c_{i}},
$$

where index $v$ represents the volume nodes of the flow grid, and $\boldsymbol{A}_{\boldsymbol{c} v}$ a $n_{v} \times n_{c}$ matrix, described as: 


$$
A_{c v}=\left[\begin{array}{cccc}
\Phi_{11} & \Phi_{12} & \cdots & \Phi_{1 \mathbf{n}_{c}} \\
\Phi_{21} & \Phi_{22} & \cdots & \Phi_{2 \mathbf{n}_{c}} \\
\vdots & \vdots & \vdots & \vdots \\
\Phi_{\mathbf{n}_{v} 1} & \Phi_{\mathbf{n}_{v} 2} & \cdots & \Phi_{\mathbf{n}_{v} \mathbf{n}_{c}}
\end{array}\right]
$$

In order to overcome the increased computational cost of the method, a relatively recently developed by the authors methodology is followed, which considers the agglomeration of the adjacent boundary nodes [19]. The idea behind this strategy is the generation of successively coarser grids via the fusion of adjacent control volumes of the finer ones. The construction of the control areas of the boundary nodes is based on a node-centered scheme, where each node's control area is defined by connecting the barycenters of surrounding triangles with the midpoints of triangle edges. It is this fusion process that can be implemented on the deformed solid boundary control cells, along with an RBFs-based mesh deformation technique, to successively reduce the set of surface points and, consequently, the dimensions of equation systems to be solved.

\section{COMPUTATIONAL METHODS}

\subsection{Flow Solver}

The simulation of unsteady incompressible flow phenomena is performed with the use of a recently developed academic code, named Galatea-I $[20,29,30]$, which employs the NavierStokes equations, coupled with the artificial compressibility method [31-33]. The discretization of the governing equations is succeeded with a node-centered finite-volume scheme on unstructured hybrid computational grids, comprised of tetrahedra, prisms and pyramids $[20,29]$. The calculation of the inviscid fluxes is performed with the Roe's approximate Riemann solver [34], along with a second-order spatial accurate scheme based on the MUSCL approach [35]. For the calculation of the viscous fluxes the gradients of velocity components have to be evaluated at the interfaces of the control cells. Two methodologies have been incorporated in Galatea-I; an element-based approach, utilizing the edge-dual control volume scheme and a simpler nodalaveraging one [20, 29]. For turbulence prediction the RANS (Reynolds-Averaged Navierstokes) approach is implemented, along with the SST (Shear Stress Transport) turbulence model [36]. At the inlet and outlet regions characteristics-based boundary conditions are applied [30], while at the solid walls free-slip or no-slip conditions can be applied depending on the type of the encountered flow (inviscid or viscous). For the time accurate computation a dual-time stepping scheme has been included [31]. According to this scheme the relaxation of the governing equations is achieved with a four-stage Runge-Kutta (RK(4)) method over pseudo-time [37], while the time accurate solution is obtained with the use of a second-order finite-difference scheme over real-time [31]. Besides using edge-based data structures and a local time-stepping technique [35], Galatea-I solver has incorporated the capability of parallel processing, based on the domain decomposition approach [20], and the implementation of an agglomeration multigrid scheme, based on the FAS (Full Approximation Scheme) approach [20], for the acceleration of the solution procedure. With the first method the initial computational grid is divided into smaller sub-domains, each of which is attributed on a single computer core [20]. Interaction between neighboring partitions is achieved with the MPI (Message Passing Interface) library functions. With the latter methodology successively coarser grids are generated, derived by the fusion of adjacent control volumes. The relaxation of the governing equations is performed on all meshes in a V-cycle type that accelerates solution convergence [20]. 


\subsection{Structural Solver}

As far as the structural computations are concerned, the open-source software package Calculix [21] is utilized, which employs Finite-Element Method (FEM) for linear or non-linear calculations on structured or unstructured three-dimensional computational grids. The solver has the capability to encounter a variety of mechanical, thermal, coupled thermo-mechanical and contact problems in a serial or parallel computational environment; for the latter one either MPI (Message Passing Interface) or OpenMP library functions can be used.

\section{NUMERICAL RESULTS}

The assessment of the pre-described methodologies was performed with the Commonwealth Advisory Aeronautical Research Council (CAARC) Standard Tall Building Model, which is in fact a specification for wind tunnel experiments on tall building geometries [22, 38]. The fullscale geometry of the building is specified as a rectangular shaped prism with base size equal to $100 \mathrm{ft}(30.8 \mathrm{~m})$ by $150 \mathrm{ft}(45.72 \mathrm{~m})$ and height $600 \mathrm{ft}(183.88 \mathrm{~m})$. It is described as a flat-topped building without parapets; its external walls are flat without any geometric disturbances [22]. The case has been encountered by several researchers, who have performed numerical tests of wind flow around the building, as well as FSI simulations [23, 39-41]; the proposed geometry has been tested in wind tunnel experiments too $[22,42]$. A sketch of the geometric model that was prepared for use with the Galatea-I solver is shown in Figure 1.

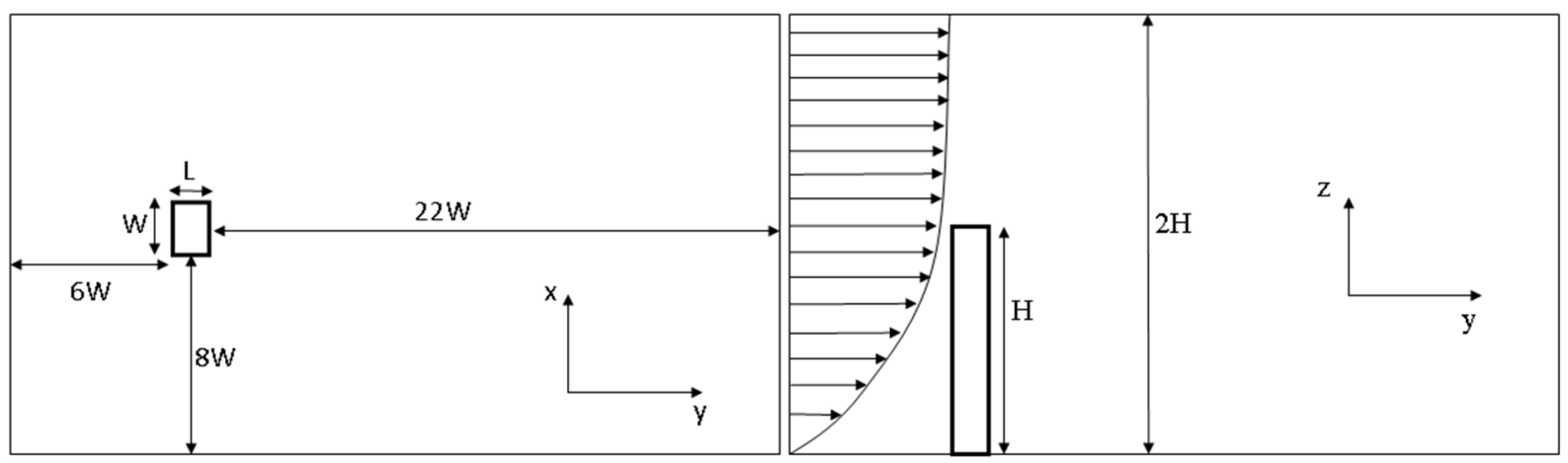

Figure 1: Sketch of CAARC test case setup.

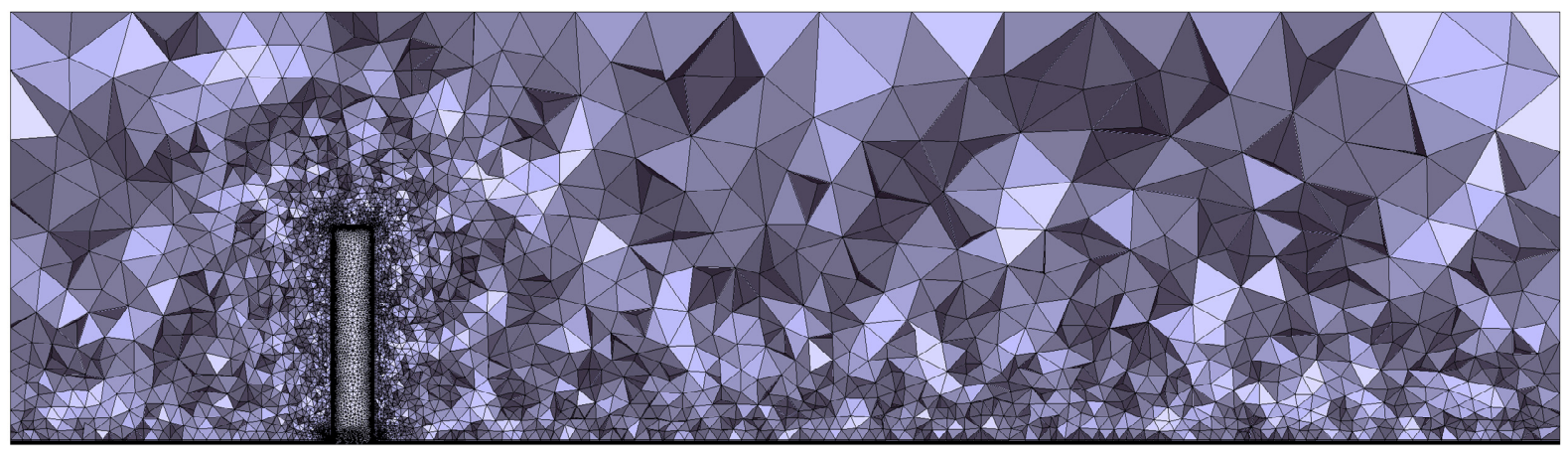

Figure 2: Computational mesh around the CAARC standard tall building model.

The computational mesh, generated for the simulation of wind flow around the CAARC building, is presented in Figure 2. As the flow around the building develops in three-dimensional fashion and is unsteady in nature, the whole building geometry had to be meshed. In order to reduce computation time a relatively coarse mesh was generated that consisted of 434,082 nodes, 623,801 tetrahedra and 640,685 prisms. Prismatic inflation was applied on the 
building walls, as well as at the base of the geometry that represents the ground around the building, in order no slip boundary conditions to be applied at these areas and the corresponding boundary layer to be effectively predicted. The prismatic inflation consisted of 20 layers with the first layer height equal to W/2500. At the top and side walls of the geometry free slip conditions were imposed.

The Reynolds number was set equal to 380,000 , based on the building height $\mathrm{H}$ and the velocity $\mathrm{c}_{\mathrm{H}}$ at height $\mathrm{z}=\mathrm{H}$ [40]. In order to simulate wind flow at such a low height, the velocity profile at the inlet was defined by the following relation

$$
\frac{|\vec{c}|}{c_{H}}=\left(\frac{z}{H}\right)^{a},
$$

where $\alpha$ is the power law exponent, which in this case was set equal to 0.3 . Moreover, the turbulence boundary conditions had to be calculated, based on the turbulence intensity in the approaching flow, as it has a significant effect on the accuracy of the numerical results [23, 39, 40]. In Figure 3 the velocity profile at the inlet, as well as the turbulence intensity profile are presented. The values of turbulence intensity at the inlet of the computational domain were interpolated from those presented in Figure 3 [39]. Finally, all values were normalized with $\mathrm{H}$ and $\mathrm{c}_{\mathrm{H}}$ for use with the dimensionless solver.
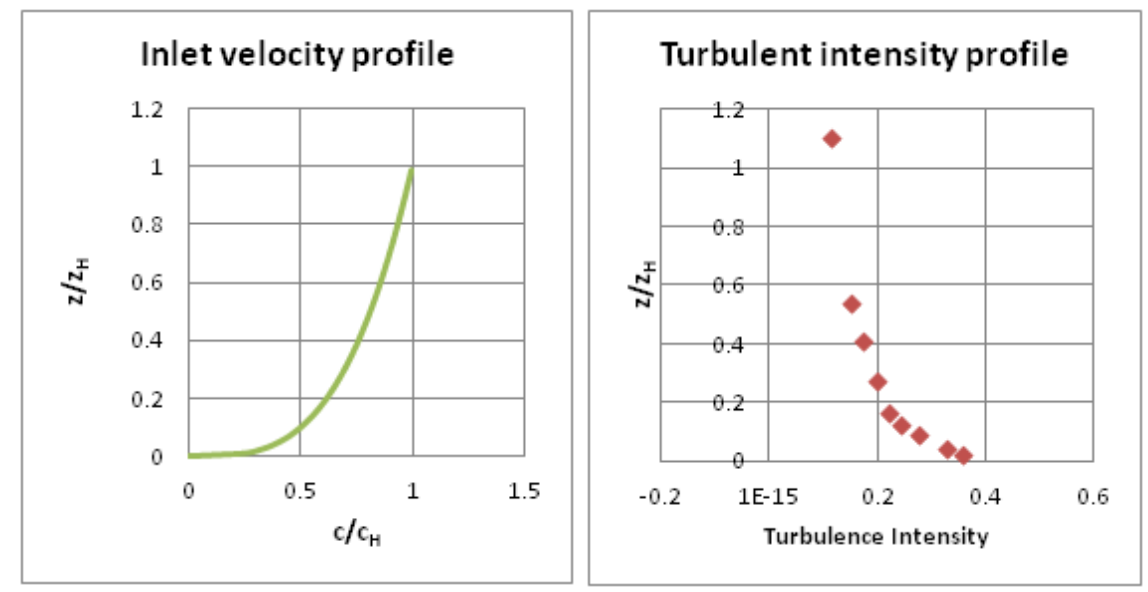

Figure 3: Inlet velocity profile (left) and turbulence intensity profile (right).

The simulation was performed on a workstation with an AMD FX 8150 Eight-Core processor at $3.62 \mathrm{Ghz}$. Acceleration via parallel processing was applied by partitioning the initial mesh into six sub-domains, while due to the small size of the computational mesh the multigrid method was not used. The time-accurate equations were solved over a total of 1300 real time iterations, with a dimensionless real time step equal to 0.05 . In each real time step 50 pseudotime iterations were performed, with a CFL number equal to 0.5 . The artificial compressibility parameter was set equal to 10.0. For the quantitative evaluation of the extracted results the mean-pressure coefficient on the wall of the building was calculated from the obtained timeaccurate results. In Figure 4 the distribution of the mean-pressure coefficient along the building wall at height $\mathrm{z}=2 / 3 \mathrm{H}$ is compared with the corresponding ones found in the literature $[39,40]$. While a very good agreement is achieved between the current and reference data at the windward and leeward sides of the building, there seems to be some disagreement between numerical and experimental results at the side of the building. This is attributed to the method of turbulence evaluation; a more accurate method (e.g., LES-Large Eddy Simulation) could provide more accurate results at these regions. 


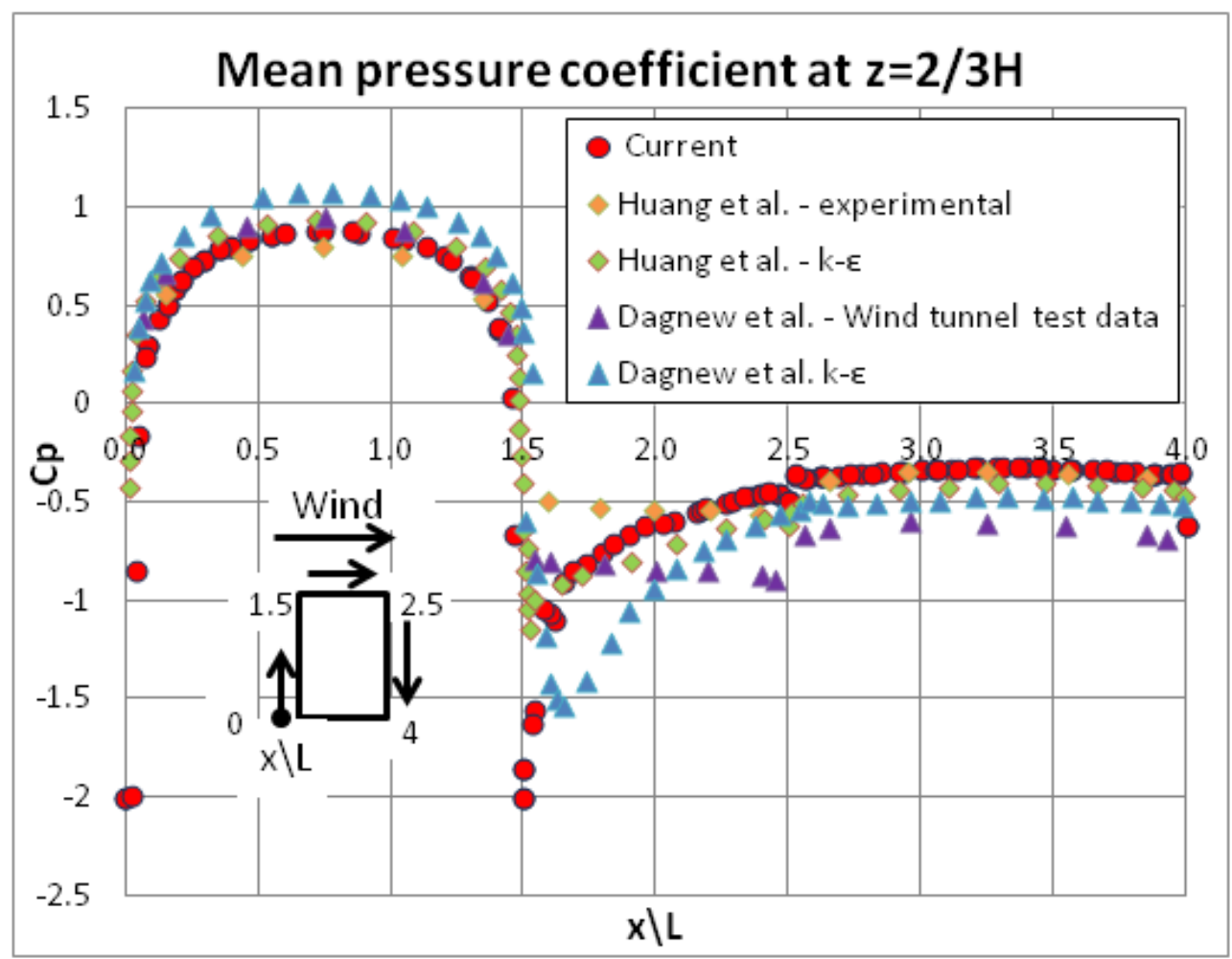

Figure 4: Mean pressure coefficient on the CAARC building wall at $\mathrm{z}=3 / 2 \mathrm{H}$.

In Figure 5 the mean pressure coefficient distribution on the building walls and the "ground" around it is presented. The maximum pressure value is located near the building top at the windward side, while under-pressure is developed at the leeward side of the building.
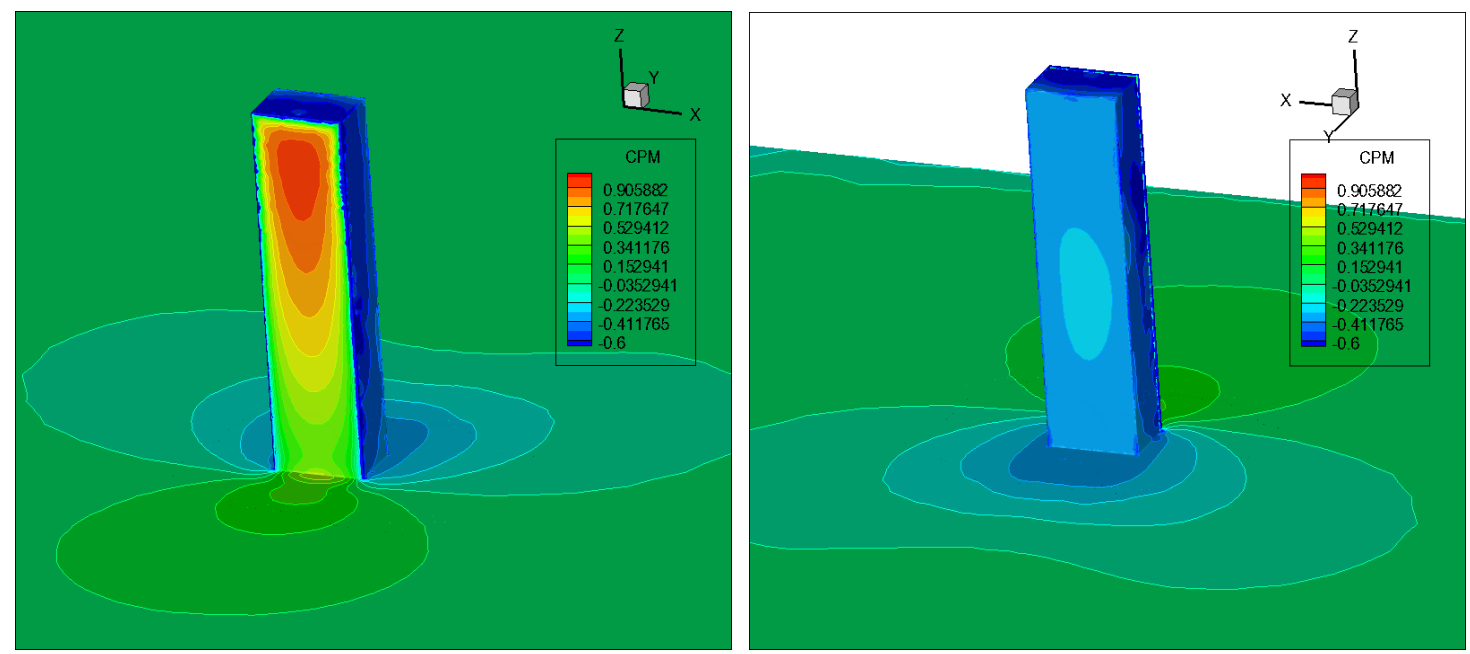

Figure 5: Mean pressure coefficient distribution on the CAARC building walls and surrounding "ground".

The force field around the CAARC standard tall building model was then calculated from the dimensionless results provided by the Galatea-I solver with regard to the flow conditions, reported by Braun et al. [23]. These properties of the flow can be found in Table 1. In order to use them with the CSD code, the exported force field had to be interpolated between the two different grids, i.e., the unstructured and the structural one. For the coupling of the two grids with the RBFs PoU method, the nearest nodes of the structural mesh have to be identified for each aerodynamic grid point at the building wall surface. Nevertheless, such an identification 
is not a straightforward procedure; a brutal linear search method would require an enormous amount of time to perform, especially in the case of very dense meshes. Therefore, custom Octree data structures were developed to find the nearest structural grid point to each aerodynamic one, while at next the remaining nodes required by the proposed methodology were gathered with the aid of the structural mesh topology. Applying this approach, whereby the number of structural points is given a priori, ensures the sparseness of the coupling matrix, as the user can specify the number of these points according to the problem needs. Due to this PoU method, a support radius needs not be defined; it is set equal to the distance of each aerodynamic grid point from the uttermost in the specific patch.

\begin{tabular}{|l|c|}
\hline Specific mass $-\rho\left[\mathrm{Kgr} / \mathrm{m}^{3}\right]$ & 1.25 \\
\hline Dynamic viscosity $-\mu\left[\mathrm{N} \cdot \mathrm{s} / \mathrm{m}^{2}\right]$ & $7.03 \cdot 10^{-2}$ \\
\hline Reference velocity $-\mathrm{c}_{\mathrm{H}}[\mathrm{m} / \mathrm{s}]$ & 100 \\
\hline Reference length $-\mathrm{H}$ & 180 \\
\hline
\end{tabular}

Table 1: Flow field properties.
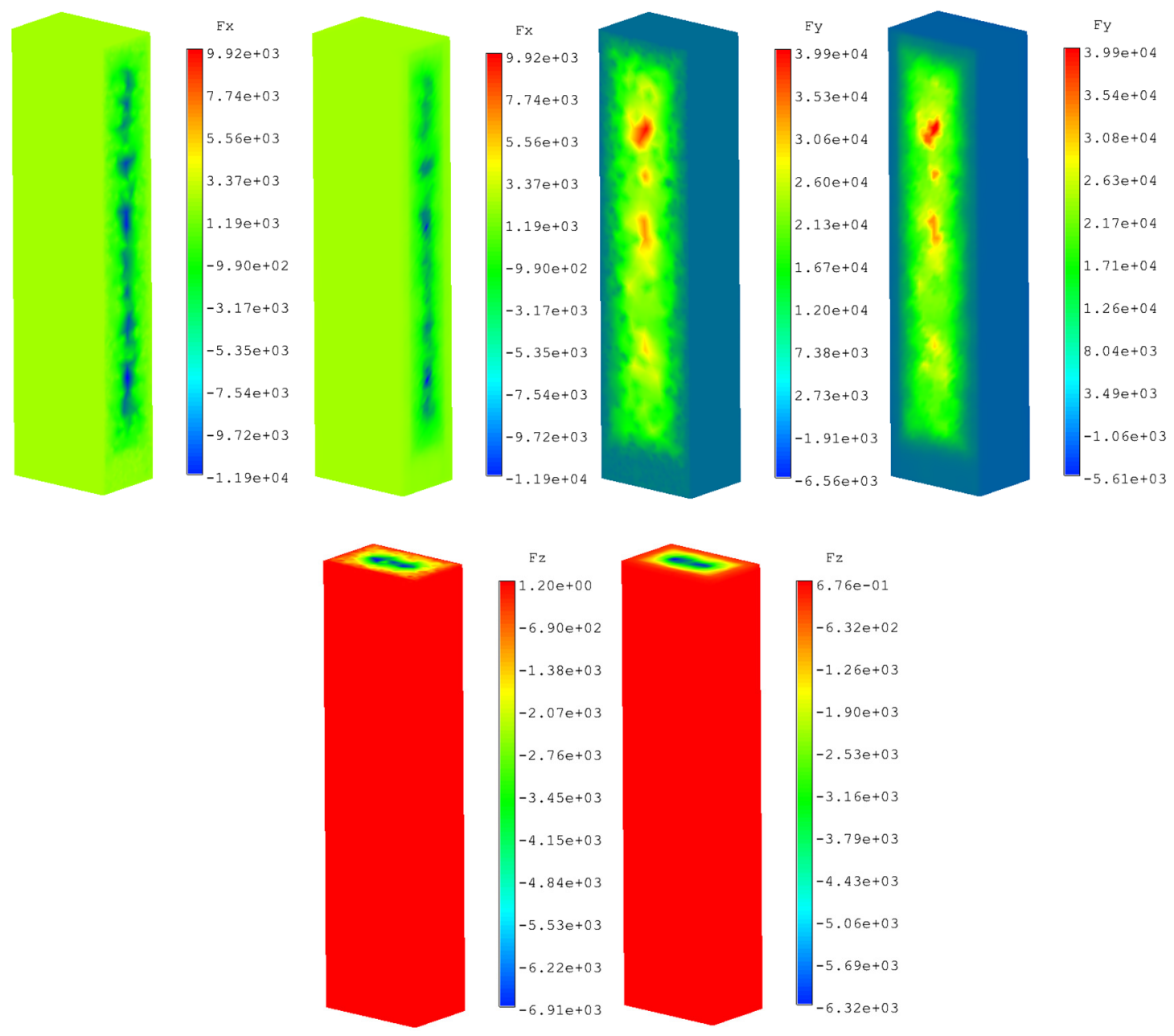

Figure 6: Comparison of force component distribution between CFD (left) and CSD (right) meshes.

The utilized structural grid is composed of 38,675 nodes and 43,048 hexahedral eight-node brick elements, fully integrated ( $2 \times 2 \times 2$ integration points). For each boundary node of the CFD mesh the 20 closest points of the CSD mesh, were used, while for each set of CSD nodes a RBFs problem was set deriving the corresponding required coefficients. While the coupling of 
the CFD with the CSD mesh may be a rather tedious procedure, it only has to be completed once (at the beginning of the FSI simulation) even for a dynamic problem. The computational cost of the coupling procedure is slightly affected by the size of the structural mesh, as the number of RBFs problems to be solved remains the same; only the nearest nodes identification process will differ. Comparisons between the force components distributions over the boundary surfaces, for the flow and structural computational grids, are illustrated in Figure 6.

These qualitative results reveal the ability of the proposed methodology to provide accurate transfer of the force field in an efficient manner though. As it can be observed, the PoU approach offers the advantage of physical distribution of forces over the interface, as opposed to the full method, where the transpose of the coupling matrix would destroy the local character of the force distribution [17].

With the loading (i.e., the force distribution) provided by the CFD code, a modal analysis was performed with the CalculiX open source software. The structural properties of the model were based on the CAARC standard tall building specifications [22], while the Young's modulus was set equal to that reported by Braun et al. [23]. All the employed structural properties are given in Table 2 .

\begin{tabular}{|l|l|}
\hline Specific mass $-\rho$ & $160 \mathrm{Kg} / \mathrm{m}^{3}$ \\
\hline Natural frequency $-\mathrm{n}$ & $0.2 \mathrm{~Hz}$ \\
\hline Young's modulus $-\mathrm{E}$ & $2.3 \cdot 10^{8} \mathrm{~N} / \mathrm{m}^{2}$ \\
\hline Poisson's ratio $-\mathrm{m}$ & 0.25 \\
\hline Structural damping $-\xi$ & $1 \%$ \\
\hline
\end{tabular}

Table 2: Mechanical properties of the structure.

In Figure 7 the von Mises stresses and the stresses at the three main axes are presented, while in Figure 8(a) the deformation of the structural mesh according to the results of the aforementioned analysis is illustrated (magnified x10) along with the un-deformed boundary surface of the fluid mesh. The maximum displacement was $46.9 \mathrm{~cm}$ on the y axis (as expected) because of the influence of the first bending mode. Moreover, in Figure 8(b) the deformed boundary surface of the CFD mesh obtained by transferring the displacement field from the CSD one, is depicted; no distortion was observed on the boundary surface of the flow grid, resulting in a smooth deformed surface. The normalized along-wind displacement $\left(\sigma_{y} / L\right)$ as a function of the reduced velocity $\left(c_{H} / n W\right)$, obtained with the proposed methodology, is compared to the corresponding results of other reference works in Table 3; a satisfactory agreement is observed.

\begin{tabular}{|l|l|}
\hline Current & 0.015633 \\
\hline Braun et al. [23] & 0.011595 \\
\hline Thepmongkorn et al. [43] & 0.013835 \\
\hline Melbourne [22] & 0.048892 \\
\hline
\end{tabular}

Table 3: Comparison of normalized maximum displacement as a function of reduced velocity. 


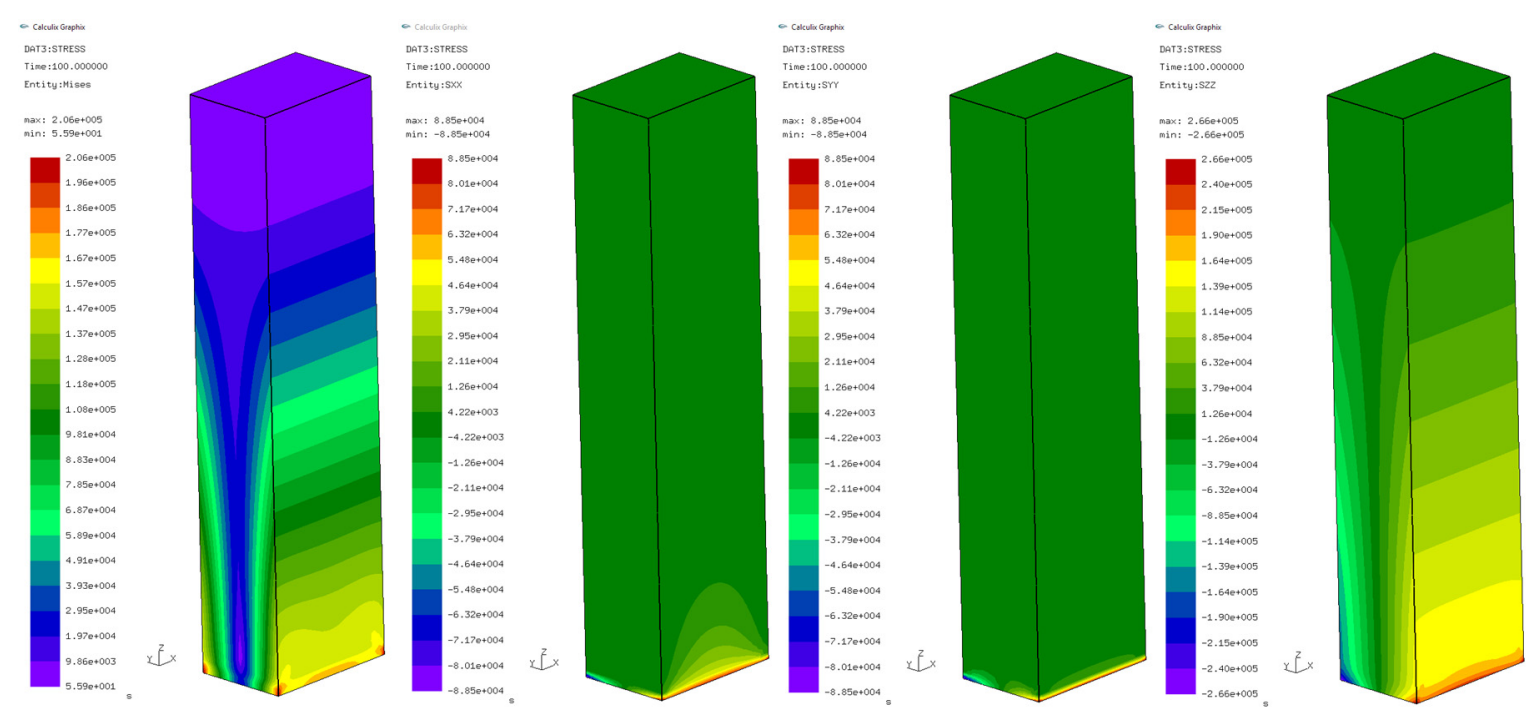

Figure 7: Von Misses, $\mathrm{S}_{\mathrm{XX}}, \mathrm{S}_{\mathrm{YY}}, \mathrm{S}_{\mathrm{ZZ}}$ stresses of the CAARC standard tall building model.

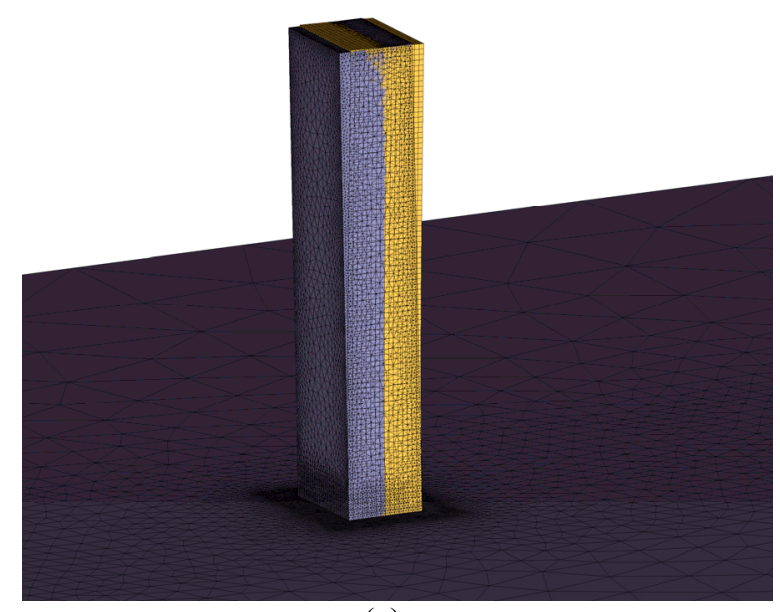

(a)

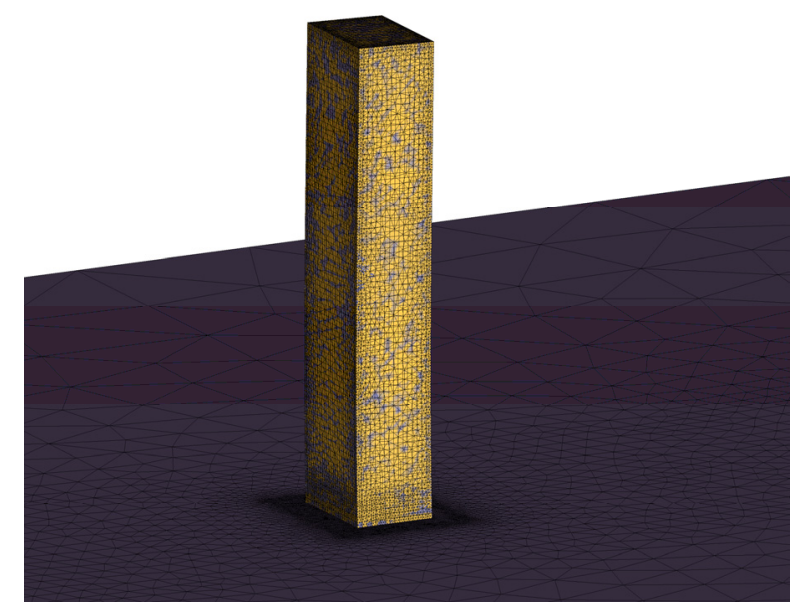

(b)

Figure 8: Deformation of the structural grid (a) and corresponding deformation of the CFD boundary grid (b).

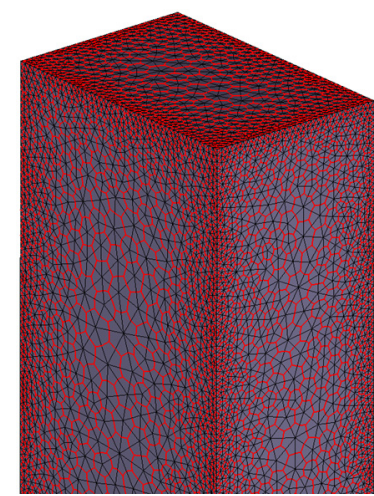

Initial $(9,724$ nodes $)$

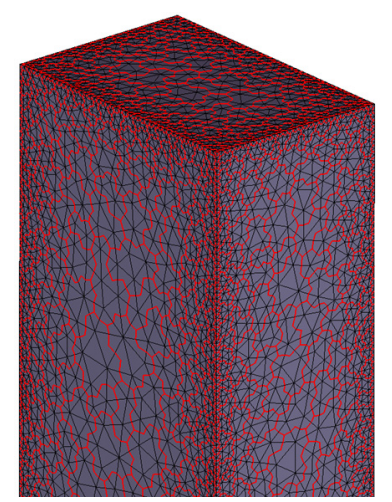

Level $1(3,494$ nodes $)$

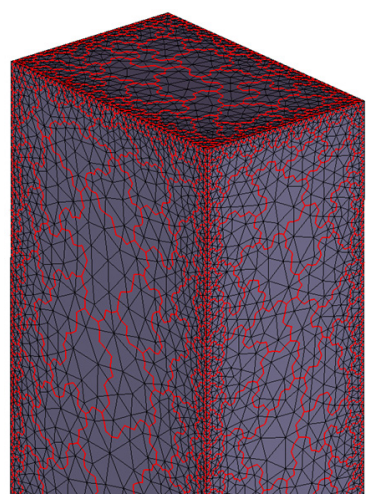

Level 2 (2,199 nodes)

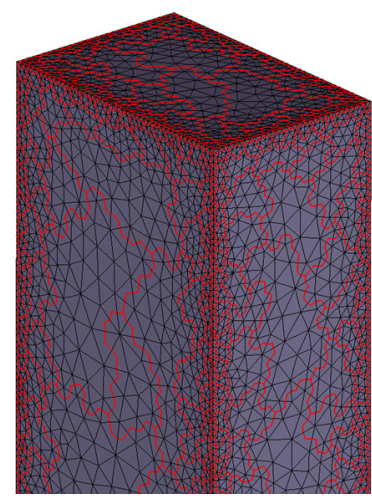

Level 3 (1,995 nodes)

Figure 9: Fine and coarser grids generated with the agglomeration procedure.

Since this stage was accomplished, the deformation of the flow grid had to be performed, according to the deflections of the boundary. For the acceleration of the procedure four coarser grids were obtained, implementing the proposed agglomeration process on the surface nodes 
[19]. In Figure 9 the boundary control areas of each level are depicted. For all of them the node with the minimum distance from their area-weighted centers is selected to be used as the base point for the RBFs method [19]. These basis points form the interpolant, required for the final deformation of the volume grid. In Figure 10, the flow grid prior and after the implementation of the deformation procedure is illustrated.

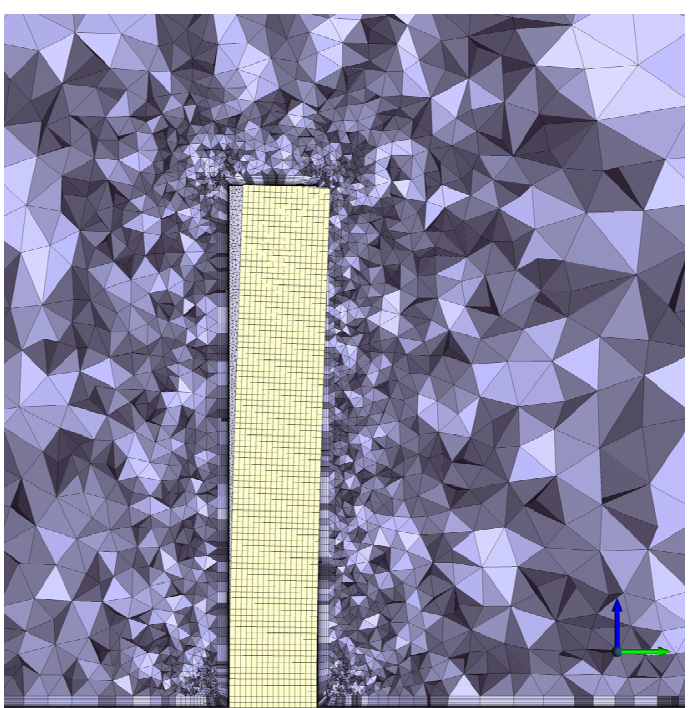

(a)

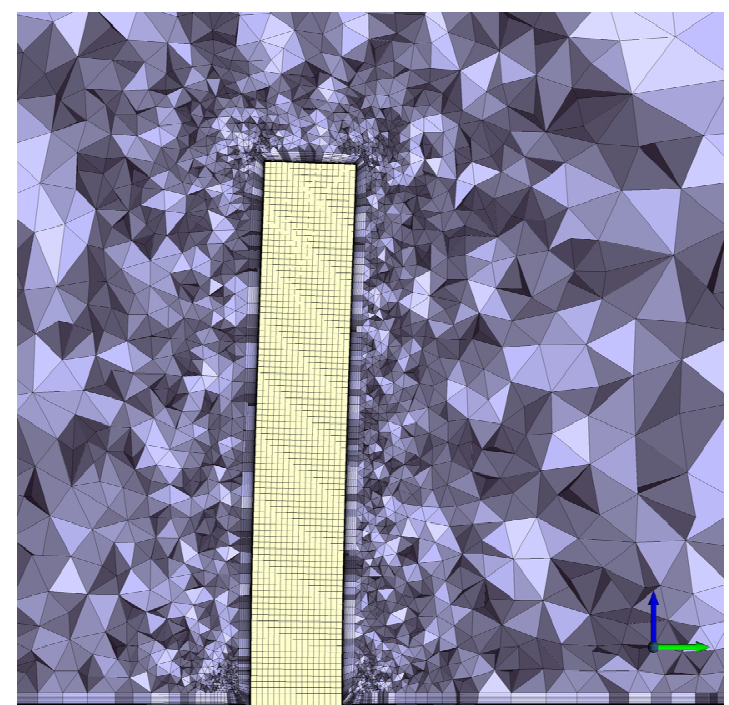

(b)

Figure 10: Before (a) and after (b) deformation states of the CFD grid.

The deformed CFD grid was subsequently used with the Galatea-I solver for a new flow simulation with the same flow conditions as with the original one. The derived mean pressure coefficient distribution around the CAARC standard tall building, along with the velocity streamlines are illustrated in Figure 11. The successfully performed simulation demonstrates the deformation methodology capabilities in providing quality grids.

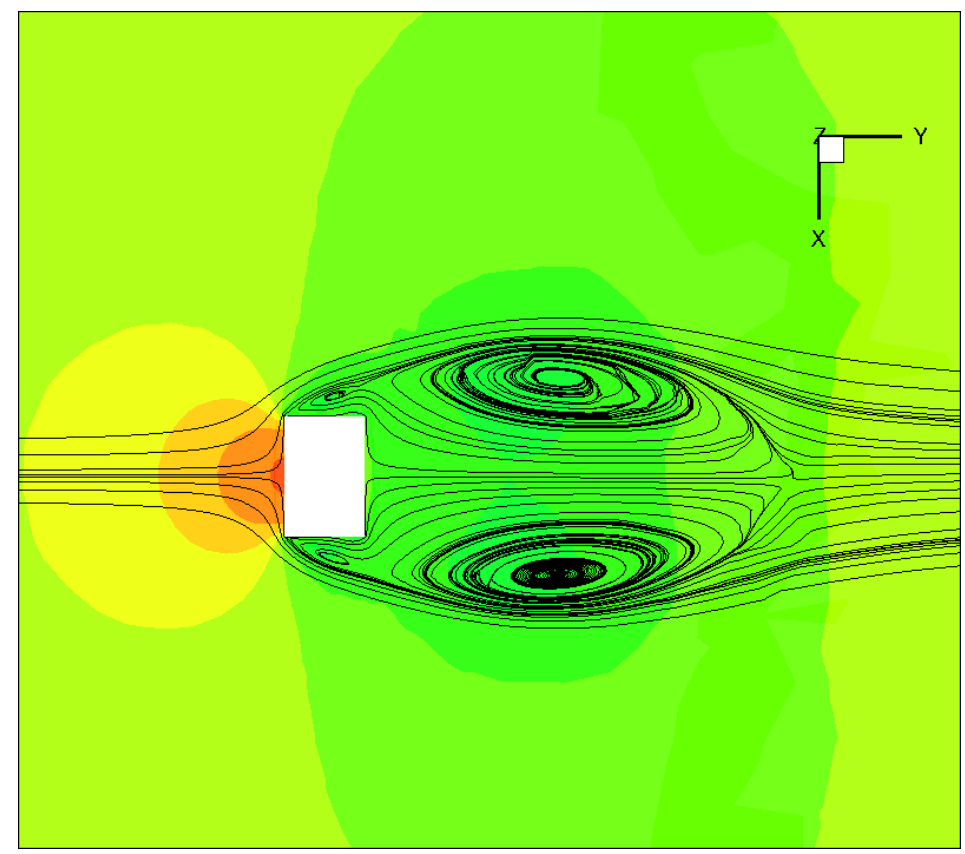

Figure 11: Mean pressure coefficient and velocity streamlines around the deformed CFD grid. 
The aforementioned analysis was based on a specification for a tall building model which however differs a lot from a realistic one. For the completion of the current work, the results from a dynamic analysis of a similar 45-storey building are presented. The building has the same external geometry as the CAARC standard tall building model, but the interior is not solid. It is consisted of beams, slabs, columns, walls, and a lift core. Moreover, two different materials, steel for the structural members (green color), and glass for all the outer walls (red color) were used (Figure 12). Table 4 presents the properties of the steel and glass material.

\begin{tabular}{|l|l|l|}
\hline & Glass & Steel \\
\hline Specific mass $-\rho$ & $2500 \mathrm{Kg} / \mathrm{m}^{3}$ & $7850 \mathrm{Kg} / \mathrm{m} 3$ \\
\hline Natural frequency $-\mathrm{n}$ & $0.2 \mathrm{~Hz}$ & $0.2 \mathrm{~Hz}$ \\
\hline Young's modulus $-\mathrm{E}$ & $7 \cdot 10^{10} \mathrm{~N} / \mathrm{m}^{2}$ & $2.1 \cdot 10^{11} \mathrm{~N} / \mathrm{m}^{2}$ \\
\hline Poisson's ratio $-\mathrm{m}$ & 0.25 & 0.25 \\
\hline Structural damping $-\xi$ & $1 \%$ & $1 \%$ \\
\hline
\end{tabular}

Table 4: Mechanical properties of the structure.

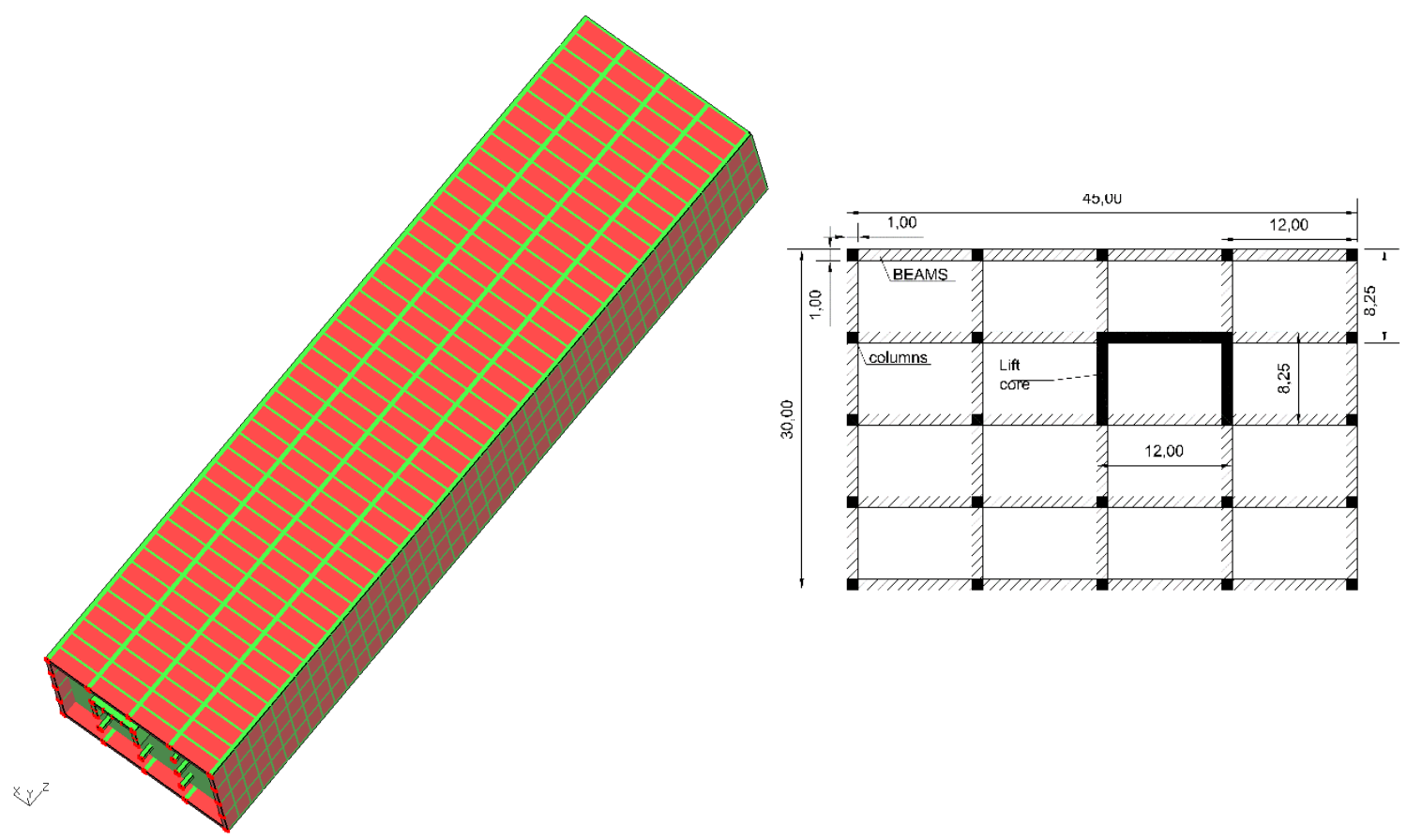

Figure 12: Realistic CAARC building model (structural members: green color, walls: red color).

In this case a dynamic analysis takes place, in which the wind loading is considered the same to the previous one (Figure 6). The deformation is also similar to the previous case but the displacements are much smaller, an expected, taking into account that the elements properties are stiffer. Figure 13 presents the extracted von Mises stresses as well as the three main stresses. Additionally, comparing to the previous problem, the stresses appear to be more realistic. The columns produce larger stresses than the walls, because of their larger stiffness, while as the height increases the stresses get smaller. In general, larger stresses were extracted (comparing to the previous case) as the same force was imposed at a smaller surface though. 

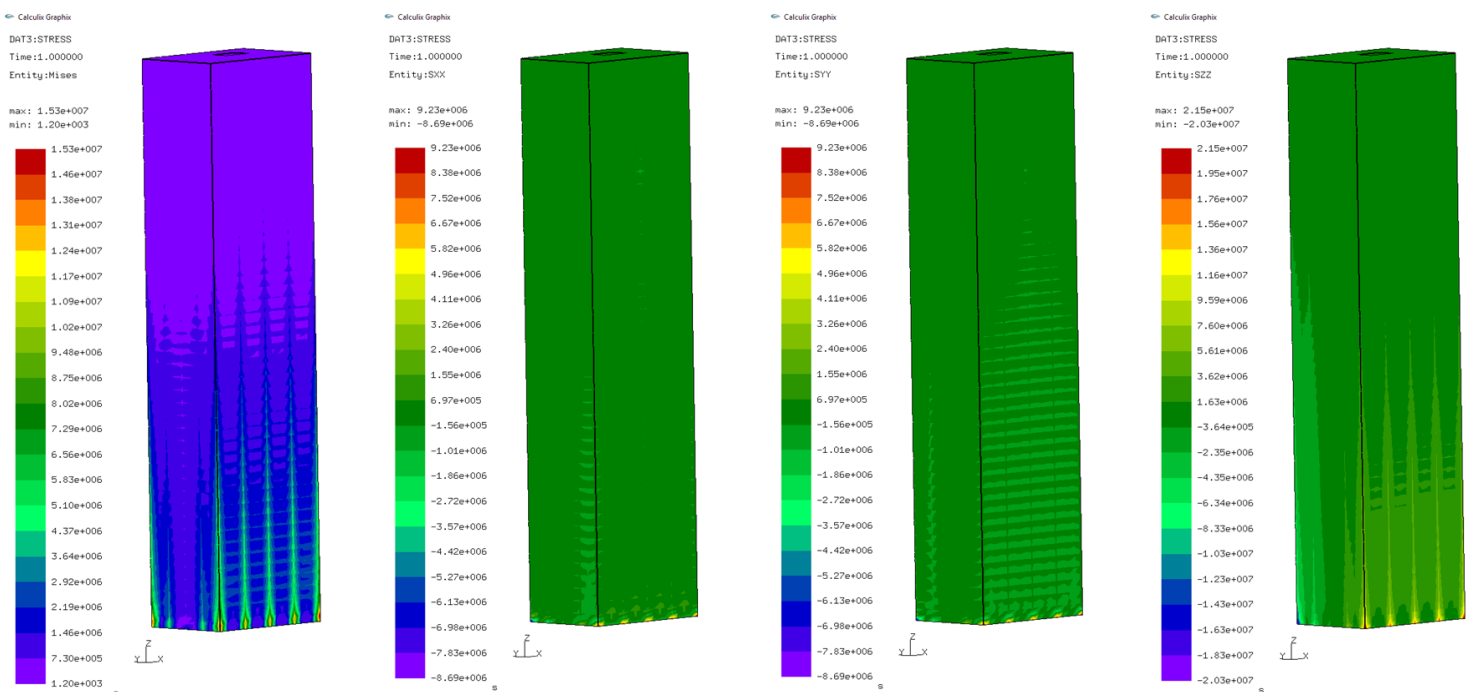

Figure 13: Von Misses, $\mathrm{S}_{\mathrm{XX}}, \mathrm{S}_{\mathrm{YY}}, \mathrm{S}_{\mathrm{ZZ}}$ stresses of the CAARC realistic building model.

\section{CONCLUSIONS}

In this paper a recently developed partitioned FSI methodology was reported. The whole procedure involved the coupling of an in-house academic CFD code, named Galatea-I [20], with the Calculix open source CSD software [21], which was succeeded employing the Radial Basis Functions (RBFs) based Partition of Unity (PoU). With this approach the conservation of energy, momentum and force is ensured over the interface of the flow and structural mesh as a result of the radial functions properties. The use of the PoU methodology improves the efficiency of the data transfer procedure providing simultaneously a physical formulation of the force distribution. The FSI methodology was applied on the CAARC standard tall building model, which was specified for similar procedures $[22,23]$. The effect of the obtained pressure field around the building was successfully transferred to the structural mesh with the PoU procedure, deriving subsequently the desired deformation; the latter agreed sufficiently with the corresponding experimental and numerical results, being available in the literature. As a result, the capabilities of the proposed methodology for such test cases in terms of accuracy and efficiency were demonstrated. A mesh deformation approach, based on the RBFs methodology but improved though by a fusion procedure, resembling the agglomeration procedure used in agglomeration multigrid methodology [19], was applied on the initial CFD mesh. The resulting deformed mesh, was subsequently imported in the Galatea-I solver, successfully providing a new flow field and hence demonstrating the deformation method's capabilities of generating quality meshes.

\section{ACKNOWLEDGMENTS}

This study was funded by Wacker Bauwerksaerodynamik GmbH, DENCO Developments \& Engineering Consultants S.A. (DENCO S.A.) and the EUREKA-EUROSTARS Project No E!7987 "Model-Based Aeroelastic Analysis of Long-Span Bridges on the HPC Cloud (BridgeCloud)"'.

\section{REFERENCES}

[1] C. F. Serge Piperno, Partitioned procedures for the transient solution of coupled aeroelastic problems part I: Model problem, theory and two-dimensional application. Computer Methods in Applied Mechanics and Engineering, 124, 79-112, 1995. 
[2] C. Farhat, M. Lesoinne, and P. LeTallec, Load and motion transfer algorithms for fluid/structure interaction problems with non-matching discrete interfaces: Momentum and energy conservation, optimal discretization and application to aeroelasticity. Computer Methods in Applied Mechanics and Engineering, 157.1, 95-114, 1998.

[3] A. Quarteroni and A. Valli, Domain decomposition methods for partial differential equations, Vol. 10. Clarendon Press, 1999.

[4] C. A. Felippa and K. C. Park, Staggered transient analysis procedures for coupled mechanical systems: Formulation. Computer Methods in Applied Mechanics and Engineering, 24, 61-111, 1980.

[5] A. de Boer, A. H. van Zuijlen, and H. Bijl, Radial basis functions for interface interpolation and mesh deformation. Advanced Computational Methods in Science and Engineering, B. Koren and K. Vuik, Eds. Springer Berlin Heidelberg, 143-178, 2009.

[6] P. Thévenaz, T. Blu, and M. Unser, Interpolation revisited. IEEE Transactions Medical Imaging, 19.7, 739-758, 2000.

[7] N. Maman and C. Farhat, Matching fluid and structure meshes for aeroelastic computations: A parallel approach. Computers \& Structures, 54.4, 779-785, 1995.

[8] P. Masarati and P. Mantegazza, A conservative mesh-free approach for fluid structure problems. Coupled Problems, 24-27, Santorini, 2005.

[9] A. de Boer, A. H. van Zuijlen, and H. Bijl, Review of coupling methods for non-matching meshes. Computer Methods in Applied Mechanics and Engineering, 196.8, 1515-1525, 2007.

[10] G. S. L. Goura, K. J. Badcock, M. A. Woodgate, and B. E. Richards, A data exchange method for fluid-structure interaction problems. Aeronautical Journal, 105, 215-221, 2001.

[11] M. Sadeghi and F. Liu, Coupled fluid-structure simulation for turbomachinery blade rows. 43rd Aerospace Sciences Meeting and Exhibit, American Institute of Aeronautics and Astronautics, Nevada, 1995.

[12] P. Chen and I. Jadic, Interfacing of fluid and structural models via innovative structural boundary element method. AIAA Journal, 36.2, 282-287, 1998.

[13] M. J. Smith, D. H. Hodges, and C. E. Cesnik, An evaluation of computational algorithms to interface between CFD and CSD methodologies. American Institute of Aeronautics and Astronautics, AIAA 96-1400, 1995.

[14] M. J. Smith, D. H. Hodges, and C. E. S. Cesnik, Evaluation of computational algorithms suitable for fluid-structure interactions. Journal of Aircraft, 37.2, 282-294, 2000.

[15] A. Beckert and H. Wendland, Multivariate interpolation for fluid-structure-interaction problems using radial basis functions. Aerospace Science and Technology, 5.2, 125-134, 2001.

[16] A. de Boer, M. S. van der Schoot, and H. Bijl, Mesh deformation based on radial basis function interpolation. Computers \& Structures, 85.11-14, 784-795, 2007.

[17] A. B. R. Ahrem, A meshless spatial coupling scheme for large-scale fluid-structure interaction problems. Computer Modeling in Engineering \& Sciences, 12.2, 2006. 
[18] T. C. S. Rendall and C. B. Allen, Improved radial basis function fluid-structure coupling via efficient localized implementation. International Journal for Numerical Methods in Engineering, 78.10, 1188-1208, 2009.

[19] G. A. Strofylas, G.N. Lygidakis, I.K Nikolos, Accelarating RBF-Based Mesh Deformation by Implementing An Agglomeration Strategy. ASME International Mechanical Engineering Congress \& Exposition (IMECE 2015), Houston, Texas, 2015, IMECE201550902.

[20] G. N. Lygidakis, S. S. Sarakinos, I. K. Nikolos. Comparison of different agglomeration multigrid schemes for compressible and incompressible flow simulations, Advances in Engineering Software, in press, 2016.

[21] G. Dhondt, CalculiX CrunchiX User's Manual, version 2.7, 2014.

[22] W. H. Melbourne. Comparison of measurements on the CAARC standard tall building model in simulated model wind flows, Journal of Wind Engineering and Industrial Aerodynamics, 6.1, 73-88, 1980.

[23] A. L. Braun, A. M. Awruch. Aerodynamic and aeroelastic analyses on the CAARC standard tall building model using numerical simulation, Computers \& Structures, 87.9, 564$581,2009$.

[24] M. H. L. Hounjet and J. J. Meijer, Evaluation of elastomechanical and aerodynamic data transfer methods for non-planar configurations in computational aeroelastic analysis. $\mathrm{Na}$ tional Aerospace Laboratory NLR, 11.1-11.24, 1995.

[25] A. Beckert, Coupling fluid (CFD) and structural (FE) models using finite interpolation elements. Aerospace Science and Technology, 4.1, 13-22, 2000.

[26] H. Wendland, Scattered data approximation, Cambridge University Press, 2004.

[27] S. Jakobsson and O. Amoignon, Mesh deformation using radial basis functions for gradient-based aerodynamic shape optimization. Computers \& Fluids, 36.6, 1119-1136, 2007.

[28] T. C. S. Rendall and C. B. Allen, Efficient mesh motion using radial basis functions with data reduction algorithms. Journal of Computational Physics, 228.17, 6231-6249, 2009.

[29] G. N. Lygidakis, S. S. Sarakinos, I. K.Nikolos. A parallel agglomeration multigrid method for incompressible flow simulations, Proceedings of the 9th International Conference on Engineering Computational Technology (ECT 2014), Naples, Italy, 2014.

[30] S.S. Sarakinos, G.N. Lygidakis, I.K. Nikolos, Evaluation of a parallel agglomeration multigrid finite-volume algorithm, named Galatea-I, for the simulation of incompressible flows on 3D hybrid unstructured grids, Proceedings of the International Mechanical Engineering Congress and Exposition (IMECE 2014), Montreal, Canada, 2014, IMECE2014-39759.

[31] Y. Kallinderis, H.T. Ahn. Incompressible Navier-Stokes method with general hybrid grids, Journal of Computational Physics, 210, 75-108, 2005.

[32] A.J. Chorin. A numerical method for solving incompressible viscous flow problems," Journal of Computational Physics, 2, 12-26, 1967.

[33] W.W. Kim, S. Menon, An unsteady incompressible Navier-Stokes solver for large eddy simulation of turbulent flows. International Journal for Numerical Methods in Fluids, 31, 983-1017, 1999. 
[34] P. Roe. Approximate Riemann solvers, parameter vectors and difference schemes, Journal of Computational Physics, 43, 357-371, 1981.

[35] J. Blazek, Computational Fluid Dynamics: Principles and Applications, Elsevier Science, Kidlington, 2001.

[36] F.R. Menter, Two-Equation Eddy-Viscosity Turbulence Models for Engineering Applications, AIAA Journal, 32, 1598-1605, 1994.

[37] M.H. Lallemand, Etude de Schemas Runge-Kutta a 4 pas pour la Resolution Multigrille des Equations d' Euler 2D, Raport de Recherche, INRIA, 1988.

[38] R. L. Wardlaw, G. F. Moss. A standard tall building model for the comparison of simulated natural winds in wind tunnels, Report CC-662 Tech25, 1970.

[39] S. Huang, Q. S. Li, S. Xu. Numerical evaluation of wind effects on a tall steel building by CFD, Journal of Constructional Steel Research, 63.5, 612-627, 2007.

[40] A. K. Dagnew, G. T. Bitsuamalk, R. Merrick. Computational evaluation of wind pressures on tall buildings. 11th American conference on Wind Engineering, San Juan, Puerto Rico, 2009.

[41] A. K. Dagnew, G. T. Bitsuamlak. LES evaluation of wind pressures on a standard tall building with and without a neighboring building, The 5th International Symposium on Computational Wind Engineering, Chapel Hill, North Carolina, USA, 2010.

[42] E. D. Obasaju. Measurement of forces and base overturning moments on the CAARC tall building model in a simulated atmospheric boundary layer. Journal of Wind Engineering and Industrial Aerodynamics, 40.2, 103-126, 1992.

[43] S. Thepmongkorn, K. C. S. Kwok, Wind-induced responses of tall buildings experiencing complex motion. J Wind Eng Ind Aerodynamics, 90, 515-526, 2002. 